\title{
CAPACIDADE DE COMBINAÇÃO ENTRE LINHAGENS DE MILHO VISANDO À PRODUÇÃO DE MILHO VERDE ( ${ }^{1}$ )
}

\author{
FABRICIO RODRIGUES $\left({ }^{2}\right)$; RENZO GARCIA VON PINHO $\left(3^{*}\right)$; CARLOS JULIANO BRANT ALBUQUERQUE $\left({ }^{4}\right)$; \\ EDMIR MARQUES FARIA FILHO $\left({ }^{5}\right)$; JEANNE DE CÁSSIA GOULART $\left({ }^{2}\right)$
}

\begin{abstract}
RESUMO
No Brasil, há escassez de informações sobre a avaliação e obtenção de cultivares destinadas à produção de milho verde, bem como estudos genéticos de características envolvidas nesta produção. Deste modo, este trabalho teve por objetivo determinar o tipo de ação gênica predominante no controle das características e estimar a capacidade geral de combinação (CGC) e a capacidade específica de combinação (CEC) entre linhagens endogâmicas de milho visando à produção de milho verde. As oito linhagens e os 28 híbridos foram avaliados no ano agrícola de 2004/2005, no município de Lavras e em Ijaci, ambos localizados na Região Sul de Minas Gerais. O delineamento experimental utilizado foi o de blocos casualizados com duas repetições, sendo analisadas sete características de interesse agronômico e comercial para a produção de milho verde. Os resultados permitiram inferir que o componente quadrático referente à CEC foi superior, para todas as características avaliadas. Constata-se que os efeitos não aditivos foram mais importantes que os aditivos para a variação dos genótipos, o que possibilita inferir que a heterose é importante na seleção de híbridos para a produção de milho verde. $\mathrm{Na}$ linhagem L55 ocorreu maior concentração de alelos favoráveis para o incremento das características PEC, PEE, MASSA, COR e COMP, sendo a mais indicada para a formação de novos híbridos; nas combinações L22xL77 (HS27), L44xL88 (HS48) e L22xL44 (HS24) houve maior efeito heterótico e média alta para as características PEE, PEC e COMP, simultaneamente, sendo estes híbridos mais indicados ao mercado de milho verde in natura.
\end{abstract}

Palavras-chave: Zea mays L., cruzamentos dialélicos, controle genético e consumo in natura.

\section{ABSTRACT \\ COMBINING ABILITY OF INBRED LINES OF SWEET CORN}

In Brazil, there is limited information on the evaluation and development of new genotypes for vegetable corn production and also on genetic studies of traits involved in this production. Thus, this research was conducted to estimate the relative importance of the general combining ability (GCA) and specific combining ability (SCA) in vegetable corn production. Eight inbred lines and 28 hybrids derived from them were evaluated in 2004/2005 season in two environments, in Lavras and Ijaci, State of Minas Gerais. A randomized complete block design with two replications per environment were used, and seven traits of agronomic and commercial interest for vegetable corn production were studied. The quadratic component of specific of combining ability (SCA) was greater than the general combining ability (GCA) for all the traits assessed. Non-additive genetic effects were more important than additive effects for genotypes variation, indicating that heterosis is important for hybrid selection for vegetable corn production. The inbred line L55 showed higher concentration of favorable alleles to the increase of traits PEC, PEE, MASSA, COR e COMP, being more suitable for the formation of new hybrid and the hybrids HS27, HS48 and HS24 accumulated a larger number of traits with SCA positive effect, significant and of high magnitude, indicating that those combinations presented better performance for all the traits, simultaneously, and can be recommended to the market for vegetable corn.

Key words: Zea mays L., diallel crosses, genetic effects and consumption in natura.

$\left({ }^{1}\right)$ Recebido para publicação em 18 de maio de 2007 e aceito em 4 de outubro de 2008.

$\left({ }^{2}\right)$ Mestrandos do Departamento de Biologia, Universidade Federal de Lavras, 37200-000 Lavras (MG). E-mail: fabriciorods@yahoo.com.br, Bolsista FAPEMIG; jinybio@yahoo.com.br

$\left({ }^{3}\right)$ Departamento de Agricultura, Universidade Federal de Lavras, Caixa Postal 3037, 37200-000 Lavras (MG). E-mail: renzo@ufla.br $\left({ }^{*}\right)$ Autor correspondente.

( ${ }^{4}$ ) EPAMIG/CTNM, Nova Porterinha (MG). E-mail: carlosjuliano@oi.com.br

$\left({ }^{5}\right)$ Estudante de Agronomia. E-mail: edmirfaria@yahoo.com.br 


\section{INTRODUÇÃO}

O consumo de milho verde sempre foi uma tradição no Brasil sendo comum à comercialização tanto do milho verde na forma de espigas ou grãos enlatados como de seus subprodutos (pamonha, curau e suco). Alimento rico em carboidratos, sendo considerado como alimento energético, é também fonte de óleo e fibras e fornece pequenas quantidades de vitaminas E, B1, B2 e ácido pantotênico, além de alguns minerais, como o fósforo e o potássio (MATOS et al., 2006).

Para o consumo in natura o milho é usualmente colhido com os grãos no estádio leitoso, entre 20 e 25 dias após a polinização, quando o estiloestigma das espigas estão de cor marrom (SAWAZAKI et al., 1979).

As cultivares ideais para a produção de milho verde devem proporcionar espigas grandes e com bom empalhamento, o que lhe confere maior proteção contra o ataque de pragas e doenças, que depreciam o produto. Os grãos devem ser, preferencialmente, do tipo dentado, de cor creme ou amarelo-creme; o endurecimento dos grãos deve ser relativamente lento e o pericarpo, fino (Oliveira et al., 1987).

Plantas de porte médio, resistentes ao acamamento e quebramento, pedúnculo firme, sabugo fino, claro e cilíndrico, com equilíbrio entre os teores de açúcar e amido, comprimento de espiga superior a $15 \mathrm{~cm}$ e diâmetro superior a $3 \mathrm{~cm}$ também devem ser considerados na escolha de cultivares para a produção de milho verde (BotTinı et al., 1995).

A disponibilidade de cultivares destinadas à produção de milho verde é muito escassa, havendo a necessidade de se desenvolverem novos híbridos apropriados para esse segmento. Em 2007, somente nove cultivares foram recomendadas para a produção de milho verde, dentre 275 cultivares de milho disponíveis para a comercialização (Cruz e Pereira FILHO, 2007).

Qualquer programa de melhoramento terá sucesso no desenvolvimento de novas cultivares, se for eficiente na seleção dos genitores a serem utilizados nos cruzamentos e que resultem em híbridos com fenótipos de interesse. A análise dialélica, devido ao grande número de informações que oferece ao melhorista, é freqüentemente utilizada em programas de melhoramento de diversas culturas. Dentre as metodologias, a proposta por GRIFFING (1956), permite obter estimativas da capacidade geral e específica de combinação, além de proporcionar informações sobre a concentração de genes predominantemente aditivos e não aditivos no controle das características.
Há escassez de informações no Brasil sobre a avaliação e obtenção de cultivares destinadas à produção de milho verde e também sobre estudos genéticos de características envolvidas nesta produção. Deste modo, este trabalho teve por objetivo determinar o tipo de ação gênica predominante de varias características e estimar a capacidade geral de combinação (CGC) e a capacidade específica de combinação (CEC) entre linhagens de milho visando à produção de milho verde.

\section{MATERIAL E MÉTODOS}

Foram utilizadas oito linhagens endogâmicas de milho, obtidas por meio de sucessivas autofecundações, com alto grau de homozigose e provenientes do programa de melhoramento da empresa GeneSeeds Recursos Genéticos em Milho Ltda. As linhagens foram escolhidas de acordo com a cor, textura e tamanho do grão, produção de espigas, empalhamento da espiga, altura da planta, altura de inserção da espiga, florescimento masculino e feminino e coloração do sabugo.

Foram realizados todos os cruzamentos possíveis entre as linhagens, para formar um dialelo completo. As linhagens foram representadas pela letra L e os híbridos pelas letras HS. Para facilitar o estudo e a visualização, os híbridos foram representados de acordo com os genitores cruzados para formá-los; por exemplo, o híbrido HS27 é proveniente do cruzamento entre a linhagem L22 e L77.

As oito linhagens e os 28 híbridos foram avaliados no ano agrícola de 2004/2005, em dois locais, no município de Lavras e no de Ijaci, ambos localizados na Região Sul de Minas Gerais. O delineamento experimental utilizado foi o de blocos casualizados com duas repetições, sendo avaliados 36 tratamentos (28 híbridos experimentais e 8 linhagens). As parcelas foram constituídas de quatro fileiras de $5 \mathrm{~m}$ com espaçamento de $0,8 \mathrm{~m}$ entre fileiras, sendo as duas fileiras centrais consideradas úteis para efeito de coleta de dados.

O preparo do solo foi realizado de maneira convencional, com uma aração e duas gradagens. A semeadura foi realizada manualmente, em 30 de outubro de 2004, em Lavras e 19 de dezembro de 2004, em Ijaci, utilizando-se oito sementes por metro linear. $\mathrm{O}$ desbaste foi realizado manualmente quando as plantas estavam com três a quatro folhas totalmente expandidas, deixando-se quatro plantas por metro, $\mathrm{o}$ que corresponde a 50.000 plantas ha- $^{-1}$.

As colheitas foram realizadas manualmente à medida que as espigas atingiram o "ponto de milho 
verde" no estádio leitoso, ou seja, quando os grãos das espigas das plantas de cada parcela estavam com $70 \%$ a $80 \%$ de teor de água, considerado adequado para envasamento e para consumo in natura.

As características avaliadas foram: PEE produtividade de espigas empalhadas por hectare, os dados foram obtidos pela soma do peso total de espigas com palha na área útil de cada parcela e transformado em kg ha ${ }^{-1}$; PEC - produtividade de espigas comerciais por hectare - os dados foram obtidos por meio da soma do peso das espigas despalhadas maiores que $15 \mathrm{~cm}$ e com diâmetro superior a $3 \mathrm{~cm}$ e isentas de pragas e doenças e transformado em kg ha-1; ALT - altura de plantas (m) - obtidos após o florescimento feminino, medindo-se seis plantas de cada parcela da área útil; DIAM diâmetro médio de espigas comerciais $(\mathrm{cm})$ - medido com o auxílio de um paquímetro o diâmetro médio de dez espigas comerciais tomadas ao acaso em cada parcela, sendo a média atribuída à parcela; e COMP - comprimento de espigas comerciais $(\mathrm{cm})$ - mediu-se o comprimento de dez espigas comerciais tomadas ao acaso em cada parcela, sendo a média atribuída à parcela. Para a avaliação da cor dos grãos (COR) foi utilizada uma escala de nota variando de 1 a 5: 1 creme, 2 - amarelo-claro, 3 - amarelo, 4 - amareloescuro e 5, alaranjado. A porcentagem de massa (MASSA) foi calculada pela relação entre a massa dos grãos e a massa total das espigas despalhadas da parcela.

Todas as análises estatísticas e genéticas foram realizadas com o auxílio do programa computacional GENES (CRUZ, 1997). Os dados coletados dessas características foram submetidos à análise de variância por local, conjunta envolvendo os dois locais e o agrupamento das médias foi feito pelo teste de Scott-Knott (1974). Utilizando o método 2 de Griffing (1956), foram estimadas a capacidade geral (CGC) e específica de combinação (CEC) e seus componentes quadráticos para todas as características avaliadas. O modelo estatístico genético utilizado foi o seguinte:

$$
Y_{i j}=m+g_{i}+g_{j}+s_{i j}+\bar{e}_{i j}
$$

Em que:

$\mathrm{Y}_{\mathrm{ij}}$ : observação da combinação híbrida envolvendo os genitores i e j;

$\mathrm{m}$ : média geral;

$\mathrm{g}_{\mathrm{i}}$ e $\mathrm{g}_{\mathrm{j}}$ : capacidade geral de combinação do iésimo e j-ésimo genitor, respectivamente;

$\mathrm{s}_{\mathrm{ij}}$ : capacidade específica de combinação para os cruzamentos entre os genitores de ordem i e j;

$\overline{\mathrm{e}}_{\mathrm{ij}}$ : erro experimental médio
Os componentes quadráticos foram estimados utilizando-se a seguinte fórmula:

$$
\phi g=\frac{Q_{C G C}-Q_{\text {erro }}}{(p+2)} \quad \phi s=Q_{C E C}-Q_{\text {erro }}
$$

\section{RESULTADOS E DISCUSSÃO}

Os resultados das análises de variância conjunta envolvendo os dois locais estão apresentados na tabela 1. Para todas as características avaliadas foram observadas diferenças significativas $(\mathrm{P}=0,01)$, com exceção da PEC $(P=0,05)$. Ficou constatada a diferença significativa entre os locais $(\mathrm{P}=0,01)$ para a maioria das características avaliadas, com exceção da característica COR. Para a interação genótipos $x$ locais não foram constatadas diferenças significativas para a maioria das características, somente para a PEE e PEC, ocorreu diferença significativa a $1 \%$ de probabilidade. Isso indica que para a maioria dos genótipos o comportamento foi semelhante nos dois locais, para as características avaliadas.

Para as características PEE e PEC, a interação pode ser constatada quando se compara o desempenho relativo de cada genótipo nos dois locais. Verifica-se que, em Ijaci, dentre os híbridos que se destacaram para a característica PEE, estão os híbridos HS47, HS17 e HS13. Já em Lavras, os mesmos híbridos não tiveram bom desempenho em relação aos demais (Tabela 2). Percebe-se que o mesmo ocorreu para a característica PEC, na qual os híbridos HS45, HS15 e HS47, em Ijaci, estavam entre os híbridos com o melhor desempenho e não se destacaram em Lavras (Tabela 2).

No sistema de produção de milho verde, normalmente, as espigas são transportadas até o local de beneficiamento ou de venda, na forma empalhada, para o consumo in natura. Esse processo reduz os danos físicos causados no transporte. Dessa forma, a produtividade de espigas empalhadas (PEE) é uma característica importante, que deve ser considerada na avaliação de cultivares para essa finalidade.

Em Lavras, entre o grupo de genótipos avaliados para a característica PEE, em 16 híbridos os valores foram superiores a $17.900 \mathrm{~kg} \mathrm{ha}^{-1}$. Com relação a Ijaci, os híbridos que se destacaram os valores foram maiores de $9.700 \mathrm{~kg} \mathrm{ha}^{-1}$. Esses resultados evidenciam o potencial dos híbridos avaliados, haja vista que somente um híbrido em Lavras e, seis em Ijaci, não obtiveram valores superiores às médias comumente relatadas no Brasil, que está entre 9 e $15 \mathrm{t}$ de milho verde por hectare (Pereira Filho et al., 2003). 
Tabela 1. Resumo da análise de variância conjunta envolvendo os dois locais (Lavras e Ijaci), para as características de altura de plantas (ALT), produtividade de espigas empalhadas (PEE), produtividade de espigas comerciais (PEC), porcentagem de massa (MASSA), cor dos grãos (COR), diâmetro (DIAM) e comprimento de espigas (COMP). UFLA, Lavras (MG), 2007

\begin{tabular}{|c|c|c|c|c|c|c|c|c|}
\hline \multirow{2}{*}{ F.V } & \multirow{2}{*}{ GL } & \multicolumn{6}{|c|}{$\mathrm{QM}$} & \multirow[b]{2}{*}{ COMP } \\
\hline & & ALT & PEE & PEC & MASSA & COR & DIAM & \\
\hline & $\mathrm{m}$ & & \multicolumn{2}{|c|}{$\mathrm{kg} \mathrm{ha}^{-1}$} & $\%$ & Escala & \multicolumn{2}{|c|}{$\mathrm{cm}$} \\
\hline Genótipos & 35 & $0,313^{* *}$ & $4.445 .731,36^{* *}$ & $23.888 .211,26^{*}$ & $484,97^{* *}$ & $2,49^{* *}$ & $0,52^{* *}$ & $20,42^{* *}$ \\
\hline Locais & 1 & $15,16^{* *}$ & $948.316 .286,11^{* *}$ & $376.837 .633,94^{* *}$ & $929,41^{* *}$ & $1,00^{\text {n.s }}$ & $4,47^{* *}$ & $131,71^{* *}$ \\
\hline Genótipos x Locais & 35 & $0,5^{\mathrm{n} . \mathrm{s}}$ & $44.927 .225,10^{* *}$ & $8.289 .692,69 * *$ & $92,98^{\text {n.s }}$ & $0,41^{\text {n.s }}$ & $0,12^{\mathrm{n} . \mathrm{s}}$ & $2,17^{\mathrm{n} . \mathrm{s}}$ \\
\hline Erro & 70 & 0,02 & $945.330,50$ & $1.169 .200,50$ & 40,87 & 0,38 & 0,07 & 1,19 \\
\hline Média & & 1,85 & $13.481,29$ & $5.493,01$ & 39,78 & 2,29 & 4,16 & 15,74 \\
\hline $\mathrm{CV} \%$ & & 8,43 & 11,77 & 20,67 & 16,06 & 27,02 & 6,81 & 7,47 \\
\hline
\end{tabular}

${ }^{* *} \mathrm{e}^{*}$ - significativo a $1 \%$ e $5 \%$ de probabilidade respectiviamente pelo teste $\mathrm{F}$; ${ }^{\mathrm{n}, \mathrm{s}}$ : não significativo.

Na avaliação de híbridos comercias e híbridos simples experimentais, para a produção de milho verde, AlbuQuerque (2005) relatou produtividades de espigas comerciais, para as cultivares AG 4051 e AG 1051, de $13.300 \mathrm{~kg} \mathrm{ha}^{-1}$ e $12.800 \mathrm{~kg} \mathrm{ha}^{-1}$, em Lavras e de $4.100 \mathrm{~kg} \mathrm{ha}^{-1}$ e $3.000 \mathrm{~kg} \mathrm{ha}^{-1}$, para Ijaci. Vale ressaltar que essas cultivares são as mais utilizadas para a produção de milho verde no Brasil.

Comparando estes resultados com os deste trabalho, no município de Lavras, somente os híbridos dos grupos "a" e "b" conforme o agrupamento feito pelo teste de SсOTT e KNOTT (1974), permitiram PEC acima desses valores (Tabela 2). Os híbridos HS24 e HS12 destacaram-se em Lavras, com produção de espigas comerciais superiores a $12 \mathrm{t} \mathrm{ha}^{-1}$. Resultados semelhantes foram obtidos por CARDOso et al. (2003), Monteiro et al. (2002), Pereira Filho et al. (2003) e TABOSA et al. (2000) utilizando os híbridos comerciais (AG 1051 e AG 4051) e híbridos simples experimentais. Em Ijaci, 15 híbridos simples se destacaram, porém, a produtividade foi menos expressiva, variando de 6.700 $\mathrm{kg} \mathrm{ha}^{-1}$ (HS15) a $4.500 \mathrm{~kg} \mathrm{ha}^{-1}$ (HS56) dentro daqueles pertencentes ao grupo superior.

A característica ALT tem importância quando se considera a utilização da planta após a colheita, pois, cultivares de menor porte são indicadas quando se deseja a incorporação dos restos culturais no solo, de forma que não prejudique o plantio posterior e seja possível maior número de colheitas por ano e por local (Bordallo et al., 2005). Os híbridos de maior altura, como, por exemplo, HS28, HS13 e HS48, são indicados quando se pretende utilizar os restos culturais na alimentação de bovinos (Tabela 3).

Características como COR, DIAM e COMP merecem maior atenção do melhorista, pois a aparência das espigas destinadas à alimentação humana é de fundamental importância para a sua comercialização e exercem grande influência na aceitação do produto (Pereira Filho et al., 2003). Desse modo, as cultivares devem ter cor dos grãos entre as notas 1 (creme) e 2 (amarelo-claro), diâmetro médio de espiga igual ou superior a $3 \mathrm{~cm}$ e comprimento igual ou superior a 15 $\mathrm{cm}$. Entre os genótipos avaliados, 18 para a característica cor dos grãos e 8 para o comprimento de espigas, não estavam dentro dos padrões exigidos pelo mercado de milho verde. Entretanto, em nenhum dos genótipos testados observou-se diâmetro inferior aos padrões exigidos (Tabela 3).

Para a característica DIAM, sete híbridos se destacaram, com valores superiores a $4,5 \mathrm{~cm}$ (Tabela 3). Em relação ao COMP, em 17 híbridos os valores foram superiores a $16,5 \mathrm{~cm}$. VALENTINI e SHIMOYA et al. (1998) relataram que o comprimento de espigas e a massa de espigas com palha são características importantes quando o milho verde se destina à comercialização em feiras.

O efeito da capacidade geral de combinação (CGC) em Lavras foi significativo $(P=0,05)$ para a maioria das características avaliadas (Tabela 4). Em Ijaci, a fonte de variação CGC não foi significativa para a produtividade de espigas empalhadas (PEE), porcentagem de massa (MASSA) e diâmetro das espigas (DIAM).

Com relação à fonte de variação CEC (Capacidade Específica de Combinação), o efeito também foi significativo para a maioria das características avaliadas, porém, para a cor dos grãos (COR) em Lavras, e MASSA, COR e DIAM em Ijaci, a CEC não foi significativa. Esses resultados corroboraram aos constatados por BORDALLO (2005), que também obteve significância para a CEC em várias características relacionadas com a produção de milho verde. 
Tabela 2. Produtividade de espigas empalhadas (PEE) e produtividade de espigas comerciais (PEC) de genótipos de milho avaliados em Lavras e Ijaci. UFLA, Lavras (MG), 2007

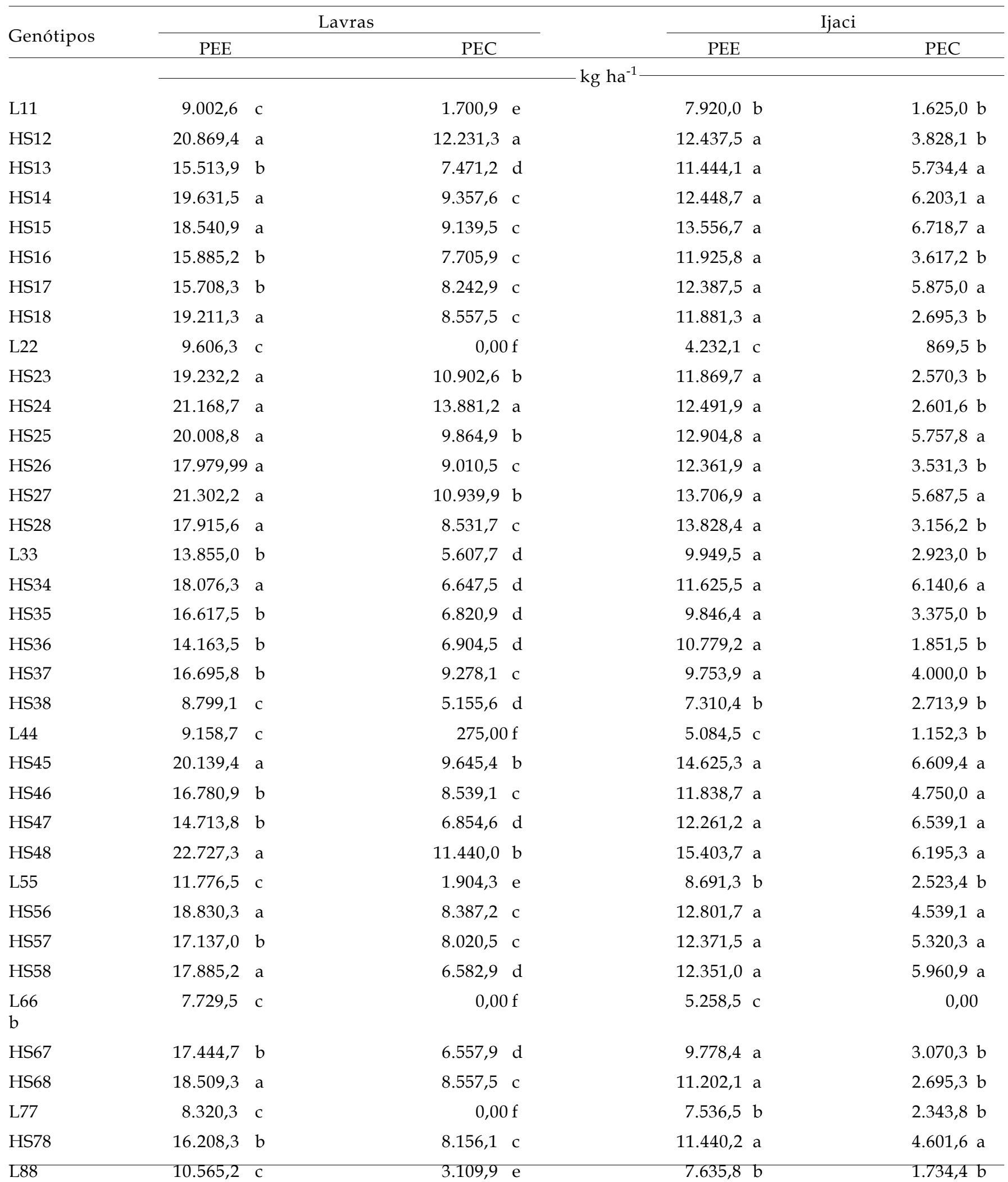

Médias seguidas pela mesma letra na coluna pertencem ao mesmo agrupamento, pelo teste de Scott-Knott, a 5\% de probabilidade. 
Tabela 3. Valores médios da altura de plantas (ALT), porcentagem de massa (MASSA), cor dos grãos (COR), diâmetro (DIAM) e comprimento das espigas (COMP), considerando a média das características em dois locais (Lavras e Ijaci). UFLA, Lavras, (MG), 2007

\begin{tabular}{|c|c|c|c|c|c|c|c|c|}
\hline Genótipos & $\mathrm{AL}$ & & MASSA & COR & DIAI & & $\mathrm{COM}$ & \\
\hline & $\mathrm{m}$ & & $\%$ & escala & 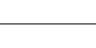 & 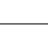 & 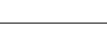 & \\
\hline L11 & 1,89 & $\mathrm{a}$ & $37,50 \mathrm{~b}$ & $2 \mathrm{~b}$ & 3,52 & c & 14,55 & $b$ \\
\hline HS12 & 2,09 & a & $41,13 \mathrm{~b}$ & $2 b$ & 4,55 & a & 16,98 & a \\
\hline HS13 & 2,16 & a & 49,74 a & $4 a$ & 4,25 & $b$ & 16,83 & a \\
\hline HS14 & 2,06 & a & $34,82 \mathrm{~b}$ & $2 \mathrm{~b}$ & 4,29 & $b$ & 18,33 & a \\
\hline HS15 & 2,04 & a & 46,09 a & $3 a$ & 4,40 & $b$ & 17,67 & $\mathrm{a}$ \\
\hline HS16 & 2,11 & a & $36,71 \mathrm{~b}$ & $2 \mathrm{~b}$ & 4,17 & $b$ & 16,18 & $b$ \\
\hline HS17 & 2,07 & a & 44,02 a & $3 \mathrm{~b}$ & 4,01 & $b$ & 17,00 & $\mathrm{a}$ \\
\hline HS18 & 1,98 & a & 47,17 a & $3 a$ & 4,41 & $b$ & 15,92 & $b$ \\
\hline L22 & 1,24 & c & $11,75 \mathrm{~d}$ & $2 \mathrm{~b}$ & 3,82 & c & 8,51 & e \\
\hline HS23 & 1,90 & a & $34,00 \mathrm{~b}$ & $2 \mathrm{~b}$ & 4,71 & a & 14,86 & $\mathrm{~b}$ \\
\hline HS24 & 1,92 & a & $37,88 \quad b$ & $2 \mathrm{~b}$ & 4,59 & $\mathrm{a}$ & 16,52 & $\mathrm{a}$ \\
\hline HS25 & 1,86 & a & 49,91 a & $3 \mathrm{~b}$ & 4,77 & a & 17,27 & a \\
\hline HS26 & 2,04 & $\mathrm{a}$ & 46,27 a & $3 a$ & 4,39 & $b$ & 15,98 & $b$ \\
\hline HS27 & 2,05 & a & 51,07 a & $2 b$ & 4,70 & a & 17,45 & $\mathrm{a}$ \\
\hline HS28 & 2,17 & $\mathrm{a}$ & $39,34 \mathrm{~b}$ & $3 a$ & 4,33 & $b$ & 15,91 & $\mathrm{~b}$ \\
\hline L33 & 1,48 & $\mathrm{~b}$ & $35,54 \mathrm{~b}$ & $2 \mathrm{~b}$ & 3,66 & c & 16,00 & $b$ \\
\hline HS34 & 2,00 & $\mathrm{a}$ & 47,75 a & $3 a$ & 4,25 & $b$ & 17,44 & $\mathrm{a}$ \\
\hline HS35 & 2,00 & $\mathrm{a}$ & $36,02 \mathrm{~b}$ & $4 \mathrm{a}$ & 4,08 & $b$ & 16,07 & $b$ \\
\hline HS36 & 2,02 & $\mathrm{a}$ & $37,37 \mathrm{~b}$ & $3 a$ & 4,26 & $\mathrm{~b}$ & 15,23 & $\mathrm{~b}$ \\
\hline HS37 & 1,93 & a & 46,22 a & 4 a & 4,15 & $b$ & 16,78 & $\mathrm{a}$ \\
\hline HS38 & 1,67 & $\mathrm{~b}$ & $38,71 \mathrm{~b}$ & $1 \mathrm{~b}$ & 3,56 & c & 11,55 & d \\
\hline L44 & 1,28 & c & $14,92 \mathrm{~d}$ & $2 \mathrm{~b}$ & 3,66 & c & 10,62 & d \\
\hline HS45 & 1,95 & a & 47,26 a & $3 a$ & 4,22 & $\mathrm{~b}$ & 18,91 & $\mathrm{a}$ \\
\hline HS46 & 1,86 & a & 54,90 a & $3 \mathrm{~b}$ & 4,54 & a & 16,63 & $\mathrm{a}$ \\
\hline HS47 & 1,83 & a & 49,71 a & $2 \mathrm{~b}$ & 4,23 & $\mathrm{~b}$ & 17,15 & $\mathrm{a}$ \\
\hline HS48 & 2,14 & a & 53,23 a & $2 \mathrm{~b}$ & 4,57 & a & 17,58 & a \\
\hline L55 & 1,55 & $\mathrm{~b}$ & $31,42 \mathrm{~b}$ & 4 a & 3,67 & c & 15,23 & $b$ \\
\hline HS56 & 1,85 & a & 52,27 a & 4 a & 4,35 & $\mathrm{~b}$ & 16,78 & a \\
\hline HS57 & 1,86 & a & 43,36 a & $3 a$ & 4,11 & $\mathrm{~b}$ & 17,17 & $\mathrm{a}$ \\
\hline HS58 & 2,05 & $\mathrm{a}$ & 49,91 a & $4 \mathrm{a}$ & 4,15 & $b$ & 16,37 & $b$ \\
\hline L66 & 1,24 & c & $14,38 \mathrm{~d}$ & $2 \mathrm{~b}$ & 3,50 & c & 10,91 & d \\
\hline HS67 & 1,72 & a & 28,68 c & $2 b$ & 4,19 & $b$ & 16,25 & b \\
\hline HS68 & 1,97 & $\mathrm{a}$ & 45,97 a & $3 \mathrm{~b}$ & 4,31 & $b$ & 15,89 & $b$ \\
\hline L77 & 1,17 & c & $20,00 \mathrm{~d}$ & $2 \mathrm{~b}$ & 3,56 & c & 12,91 & c \\
\hline HS78 & 1,91 & a & $42,06 \mathrm{~b}$ & $2 \mathrm{~b}$ & 4,08 & $\mathrm{~b}$ & 16,92 & $\mathrm{a}$ \\
\hline L88 & 1,46 & $\mathrm{~b}$ & $34,79 \mathrm{~b}$ & $1 \mathrm{~b}$ & 3,69 & c & 14,29 & b \\
\hline Média & 1,85 & & 39,77 & 2,47 & 4,16 & & 15,74 & \\
\hline
\end{tabular}

Médias seguidas pela mesma letra na coluna pertencem ao mesmo agrupamento, pelo teste de Scott-Knott, a 5\% de probabilidade. 
A significância da CGC e CEC para as características ALT, PEE, COR, DIAM e COMP no desdobramento da fonte genótipos na análise dialélica conjunta está de acordo com os resultados observados por Lemos et al. (2002) e Teixeira et al. (2001) evidenciando a existência de diferenças significativas entre os genótipos tanto para os efeitos gênicos aditivos como os não-aditivos (Tabela 4).

Considerando a análise dialélica conjunta, verifica-se que o quadrado médio da CGC não foi significativo para as características MASSA e PEC (Tabela 4). A CEC foi significativa para todas as características avaliadas no experimento, indicando que há híbridos formados a partir do cruzamento de linhagens com melhor desempenho que outros, devido aos efeitos não aditivos envolvidos no controle da característica.

A significância do efeito da interação CGC $x$ Locais para as características PEC, PEE e COMP, revela que linhagens parentais não exibem o mesmo comportamento "per se" e suas frequências alélicas diferem para essas características. Demonstra também que as linhagens contribuem de forma diferente na expressão dessas características nos dois locais.

Como o efeito da interação da CEC $x$ Locais foi significativo $(\mathrm{P} \leq 0,05)$ para $\mathrm{PEE}$ e $\mathrm{PEC}$, denota-se que os cruzamentos são heterogêneos e diferem do comportamento esperado com base na capacidade geral de combinação de seus parentais e também que a heterose obtida pelos híbridos variou nos dois locais (Tabela 4).

Tabela 4. Desdobramento do efeito de genótipos em capacidade geral de combinação (CGC), capacidade específica de combinação (CEC) e interações CGC x Locais e CEC x Locais, considerando as análises dialélicas feitas por local e a análise dialélica conjunta envolvendo os dois locais. UFLA, Lavras (MG), 2007

\begin{tabular}{|c|c|c|c|c|c|c|c|c|}
\hline \multirow{2}{*}{ Fonte de variação } & \multirow{2}{*}{ GL } & \multicolumn{7}{|c|}{$\mathrm{QM}$} \\
\hline & & ALT & PEE & PEC & MASSA & $\mathrm{COR}$ & DIAM & COMP \\
\hline & \multirow[t]{2}{*}{$\mathrm{m}$} & & $\mathrm{kg}$ & $a^{-1}$ & $\%$ & Escala & $\bar{c}$ & \\
\hline & & \multicolumn{7}{|c|}{ Lavras } \\
\hline CGC & 7 & $0,20^{*}$ & $14.860 .124,17^{* *}$ & $7.163 .733,41^{* *}$ & $276,51^{*}$ & $2,75^{*}$ & $0,38^{* *}$ & $3,68^{\text {n.s }}$ \\
\hline \multirow[t]{2}{*}{ CEC } & 28 & $0,23^{* *}$ & $39.513 .561,95^{* *}$ & $29.831 .276,20^{* *}$ & $298,95^{* *}$ & $1,22^{\text {n.s }}$ & $0,40^{* *}$ & $12,17^{* *}$ \\
\hline & & \multicolumn{7}{|c|}{ Ijaci } \\
\hline CGC & 7 & $0,94^{* *}$ & $4.648 .231,36^{\text {n.s }}$ & $9.091 .183,83^{*}$ & $123,81^{\text {n.s }}$ & $3,20^{* *}$ & $0,18^{\text {n.s }}$ & $17,56^{* *}$ \\
\hline \multirow[t]{2}{*}{ CEC } & 28 & $0,15^{* *}$ & $16.797 .169,74^{* *}$ & 6.327.374,42* & $323,41^{\mathrm{n} . \mathrm{s}}$ & $0,93^{\text {n.s }}$ & $0,26^{\text {n.s }}$ & $10,76^{* *}$ \\
\hline & & \multicolumn{7}{|c|}{ Conjunta } \\
\hline CGC & 7 & $0,23^{* *}$ & $15.370 .568,16^{*}$ & $8.272 .832,04^{\text {n.s }}$ & $232,77^{n . s}$ & $5,52^{* *}$ & $0,42^{*}$ & $16,34^{*}$ \\
\hline CEC & 28 & $0,33^{* *}$ & $51.729 .272,16^{* *}$ & $27.792 .056,06^{*}$ & $548,02^{* *}$ & $1,73^{* *}$ & $0,55^{* *}$ & $21,44^{* *}$ \\
\hline CGC $\times$ Locais & 7 & $0,06^{\text {n.s }}$ & $4.137 .787,37^{* *}$ & $7.982 .085,21^{* *}$ & $167,55^{\text {n.s }}$ & $0,43^{\text {n.s }}$ & $0,14^{\text {n.s }}$ & $4,89^{*}$ \\
\hline CEC $x$ Locais & 28 & $0,05^{\mathrm{n} . \mathrm{s}}$ & $4.581 .459,53^{* *}$ & $8.366 .597,56^{* *}$ & $74,34^{\text {n.s }}$ & $0,41^{\mathrm{n} . \mathrm{s}}$ & $0,12^{\text {n.s }}$ & $1,48^{\text {n.s }}$ \\
\hline
\end{tabular}

${ }^{*} \mathrm{e}^{* *}$ - significativo a de $1 \%$ e $5 \%$ de probabilidade respectivamente pelo teste $\mathrm{F}$. ${ }^{\mathrm{n}, \mathrm{s}}$ : não significativo.

O componente quadrático referente a $\operatorname{CEC}\left(\phi_{s}\right)$ obtido na análise conjunta, foi 35 vezes superior para a característica PEE e 37 vezes para PEC ao componente quadrático referente à CGC $\left(\phi_{g}\right)$. Com relação à MASSA esse valor foi superior em 26 vezes, para COR três vezes e 13 vezes superior para as características ALT, DIAM e COMP. Por esses valores, constata-se que os efeitos gênicos não aditivos prevaleceram aos aditivos, confirmando os resultados obtidos por SCAPIM et al. (1995).

Com relação aos efeitos da capacidade geral de combinação $\left(g_{i}\right)$, a linhagem L11 revelou valor positivo e significativo para a característica ALT, o que indica tendência de aumento na altura da planta para os cruzamentos em que a linhagem participa (Tabela 5).
Para a característica DIAM, o efeito de $g_{i}$ das linhagens L22 e L44 superaram as demais. Para a característica COMP, nas linhagens L11 e L55 os valores foram superiores às demais, visto que essas linhagens possuem maior concentração de alelos favoráveis para o incremento dessas características.

A cor dos grãos dos híbridos HS12, HS16, HS17, HS47, HS27 e HS48 é amarelo-creme, considerada a de melhor aparência para a comercialização in natura das espigas. Verifica-se que nesses híbridos não estão envolvidas as linhagens L33 e L55, que têm efeito de $g_{i}$ positivo e significativo para essa característica (Tabela 5). 
Tabela 5. Estimativas dos efeitos médios da capacidade geral de combinação $\left(g_{i}\right)$ para sete características obtidas a partir de 36 genótipos, avaliados em dois locais, em esquema de cruzamento dialélico completo envolvendo oito linhagens. UFLA, Lavras (MG), 2007

\begin{tabular}{|c|c|c|c|c|c|c|c|}
\hline Genótipos & ALT & PEE & PEC & MASSA & COR & DIAM & COMP \\
\hline & $\mathrm{m}$ & \multicolumn{2}{|c|}{$\mathrm{kg} \mathrm{ha}^{-1}$} & $\%$ & Escala & \multicolumn{2}{|c|}{$\mathrm{cm}$} \\
\hline L11 & $0,166^{* *}$ & $131,17^{* *}$ & $257,78^{\mathrm{n} . \mathrm{s}}$ & $1,67^{\text {n.s }}$ & $-0,137^{*}$ & $-0,031^{n . s}$ & $0,635^{* *}$ \\
\hline L22 & $-0,013^{n . s}$ & $654,57^{* *}$ & $268,09^{n . s}$ & $-3,49 * *$ & $-0,212^{*}$ & $0,225^{* *}$ & $-0,97 * *$ \\
\hline L33 & $0,001^{\mathrm{n} . \mathrm{s}}$ & $-666,33^{*}$ & $-112,34^{\text {n.s }}$ & $0,29^{n . s}$ & $0,188^{*}$ & $-0,083^{*}$ & $-0,089^{\text {n.s }}$ \\
\hline L44 & $-0,03^{\text {n.s }}$ & $487,42^{\text {n.s }}$ & $469,25^{* *}$ & $-0,26^{\mathrm{n} \cdot \mathrm{s}}$ & $-0,237^{* *}$ & $0,059^{*}$ & $0,213^{n . s}$ \\
\hline L55 & $0,008^{\mathrm{n} . \mathrm{s}}$ & $794,42^{* *}$ & $336,19^{*}$ & $2,97^{* *}$ & $0,813^{* *}$ & $-0,001^{\text {n.s }}$ & $0,904^{* *}$ \\
\hline L66 & $-0,057^{*}$ & $-820,27^{* *}$ & $-957,64^{* *}$ & $-2,70^{* *}$ & $0,113^{\text {n.s }}$ & $-0,021^{\mathrm{n} . \mathrm{s}}$ & $-0,69^{* *}$ \\
\hline L77 & $-0,092 * *$ & $-501,98^{*}$ & $-52,04^{\mathrm{n} . \mathrm{s}}$ & $-1,28^{\mathrm{n} \cdot \mathrm{s}}$ & $-0,262^{* *}$ & $-0,083^{*}$ & $0,289^{n . s}$ \\
\hline L88 & $0,017^{n . s}$ & $-79,39^{\text {n.s }}$ & $-209,28^{\mathrm{n} . \mathrm{s}}$ & $2,8^{* *}$ & $-0,262^{* *}$ & $-0,065^{\mathrm{n} . \mathrm{s}}$ & $-0,293^{n \cdot s}$ \\
\hline
\end{tabular}

${ }^{*} \mathrm{e}^{* *}$ - significativo a $5 \%$ e a $1 \%$ de probabilidade respectivamente pelo teste $t$. ${ }^{\text {n.s. }}$ : não significativo.

Para as características de PEE e PEC, as linhagens L11, L22 e L55 para PEE e as linhagens L44 e L55 para PEC possuem valores significativos, positivos e altos de gi. Esses valores atestam que essas linhagens têm maior probabilidade de formar híbridos de milho verde com fenótipos mais desejáveis, pois, o progenitor em questão é superior aos demais progenitores incluídos no dialelo, com relação ao comportamento médio dos cruzamentos.

A linhagem L55, dentre os progenitores avaliados, proporcionou maior contribuição para o aumento das características PEE, PEC, MASSA, COR e COMP, simultaneamente, e somente para cor dos grãos, o efeito positivo e significativo não é desejado (Tabela 5).

Os cruzamentos entre as linhagens L44 x L88 (HS48), L22 x L77 (HS27), L44 x L55 (HS45) e o L11 x L22 (HS12) destacaram-se pelos altos valores da capacidade específica de combinação $\left(s_{i j}\right)$ para a característica PEE, com valores de $5175 \mathrm{~kg} \mathrm{ha}^{-1}, 3870$ $\mathrm{kg} \mathrm{ha}^{-1}, 2620 \mathrm{~kg} \mathrm{ha}^{-1}$ e $2380 \mathrm{~kg} \mathrm{ha}^{-1}$, respectivamente (Tabela 6). Esses valores evidenciam a existência de heterose nos híbridos e confirmam a significância da CEC para esta característica.

Nota-se que os híbridos mais promissores para a PEC foram os HS48 (L44 x L88) e o HS24 (L22 $x$ L44) (Tabela 6). Verifica-se que nos híbridos com valores positivos e altos para o efeito sij, as linhagens utilizadas nos cruzamentos apresentaram valores para o efeito de gi positivo somente em um dos parentais.

É importante ressaltar que são desejáveis aquelas combinações híbridas com estimativas de $s_{i j}$ mais favoráveis, que envolvam os dois genitores que apresentem efeito de $g_{i}$ mais favorável, ou seja, com valor positivo e significativo. Deste modo, a combinação entre as linhagens L44 e L55 (HS45), em que se obteve efeito positivo para $s_{i j}$ e para o efeito de $g_{i}$ nas duas linhagens parentais, é a mais desejada (Tabelas 5 e 6).

Os híbridos HS28, HS27, HS48 e HS26 obtiveram os maiores valores do efeito de $s_{i j}$ para a característica ALT e os híbridos HS28, HS37 e HS13 para COR (Tabela 6). Vale ressaltar que os valores positivos de $s_{i j}$ ou até mesmo de $g_{i}$ para essas características são indesejáveis. Neste caso, o mais indicado é a utilização de híbridos que possuam valor negativo e significativo para o efeito de $s_{i j}$ e também para o efeito de CGC.

Para a característica MASSA, os cruzamentos L66 x L77 (HS67) e L33 x L55 (HS35), foram aqueles com o menor valor do efeito de $s_{i j}$, indicando ser essas combinações indesejáveis. Com relação ao DIAM e COMP, os cruzamentos L22 x L77 (HS27), L22 x L55 (HS25) e L44 x L66 (HS46) proporcionaram valores de $s_{i j}$ positivos, significativos e de grande magnitude. Comprova-se a maior possibilidade de exploração da heterose considerando simultaneamente essas duas características que são importantes no milho verde.

Os híbridos HS27, HS48, HS45 e HS24 acumularam maior número de características com efeito de $s_{i j}$ positivo, significativo e de alta magnitude, comprovando que essas combinações são as de melhor desempenho de forma simultânea para as características avaliadas e com maior exploração da heterose. 
Tabela 6. Estimativas dos efeitos médios da capacidade específica de combinação $\left(s_{i j}\right)$ de 36 genótipos para sete características de milho verde, avaliados em dois locais, em esquema de cruzamento dialélico completo envolvendo oito linhagens. UFLA, Lavras (MG), 2007

\begin{tabular}{|c|c|c|c|c|c|c|c|}
\hline Genótipos & ALT & PEE & PEC & MASSA & COR & DIAM & COMP \\
\hline & $\mathrm{m}$ & 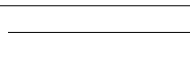 & $a^{-1}$ & $\%$ & Escala & 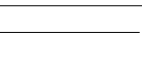 & 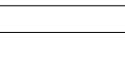 \\
\hline L11 & $-0,29 * *$ & $-5.282,33^{* *}$ & $-4.345,60^{* *}$ & $-5,61^{*}$ & $-0,52^{*}$ & $-0,58^{* *}$ & $-2,46^{* *}$ \\
\hline HS12 & $0,09^{\mathrm{n} \cdot \mathrm{s}}$ & $2.386,40^{* *}$ & $2.010,83^{* *}$ & $3,17^{\mathrm{n} . \mathrm{s}}$ & $0,06^{\mathrm{n} \cdot \mathrm{s}}$ & $0,19^{\mathrm{n} . \mathrm{s}}$ & $1,57^{* *}$ \\
\hline HS13 & $0,15^{*}$ & $532,87^{\mathrm{n} \cdot \mathrm{s}}$ & $964,36^{\text {n.s }}$ & $7,99 * *$ & $0,66^{*}$ & $0,21^{\mathrm{n} . \mathrm{s}}$ & $0,54^{\mathrm{n} \cdot \mathrm{s}}$ \\
\hline HS14 & $0,08^{\mathrm{n} . \mathrm{s}}$ & $1.939,873^{*}$ & $1.560,32^{* *}$ & $-6,36^{* *}$ & $-0,67^{*}$ & $0,11^{\mathrm{n} . \mathrm{s}}$ & $1,74^{* *}$ \\
\hline HS15 & $0,02^{n . s}$ & $1.641,593^{*}$ & $1.842,15^{* *}$ & $1,67^{\mathrm{n} . \mathrm{s}}$ & $-0,22^{\mathrm{n} \cdot \mathrm{s}}$ & $0,27^{*}$ & $0,39^{n . s}$ \\
\hline HS16 & $0,15^{*}$ & $1.113,29^{\mathrm{n} . \mathrm{s}}$ & $868,42^{\text {n.s }}$ & $-2,03^{n . s}$ & $-0,27^{\mathrm{n} . \mathrm{s}}$ & $0,06^{\mathrm{n} . \mathrm{s}}$ & $0,50^{\mathrm{n} . \mathrm{s}}$ \\
\hline HS17 & $0,15^{*}$ & $937,45^{\text {n.s }}$ & $1.360,21^{* *}$ & $3,86^{\mathrm{n} . \mathrm{s}}$ & $0,36^{\mathrm{n} . \mathrm{s}}$ & $-0,04^{\mathrm{n} \cdot \mathrm{s}}$ & $0,34^{\mathrm{n} . \mathrm{s}}$ \\
\hline HS18 & $-0,05^{* *}$ & $2.013,24^{* *}$ & $84,92^{* *}$ & $2,93^{\mathrm{n} . \mathrm{s}}$ & $1,11^{\text {n.s }}$ & $0,34^{\mathrm{n} . \mathrm{s}}$ & $-0,16^{* *}$ \\
\hline L22 & $-0,58^{* *}$ & $-7.871,21^{* *}$ & $-5.593,92^{* *}$ & $-21,05^{* *}$ & $0,13^{n . s}$ & $-0,78^{* *}$ & $-5,30^{* *}$ \\
\hline HS23 & $0,06^{\mathrm{n} . \mathrm{s}}$ & $2.081,40^{* *}$ & $1.087,71^{*}$ & $-2,58^{\mathrm{n} \cdot \mathrm{s}}$ & $-0,52^{\mathrm{n} . \mathrm{s}}$ & $0,41^{* *}$ & $0,17^{\mathrm{n} . \mathrm{s}}$ \\
\hline HS24 & $0,12^{\mathrm{n} . \mathrm{s}}$ & $2.206,66^{* *}$ & $2.011,06^{* *}$ & $1,85^{\mathrm{n} . \mathrm{s}}$ & $-0,59^{*}$ & $0,15^{\mathrm{n} . \mathrm{s}}$ & $1,54^{* *}$ \\
\hline HS25 & $0,01^{\mathrm{n} . \mathrm{s}}$ & $1.526,51^{*}$ & $1.714,06^{* *}$ & $10,65^{* *}$ & $-0,64^{*}$ & $0,38^{* *}$ & $1,59^{* *}$ \\
\hline HS26 & $0,27^{* *}$ & $1.855,35^{* *}$ & $1.467,40^{* *}$ & $12,68^{* *}$ & $0,31^{\text {n.s }}$ & $0,03^{n . s}$ & $1,89^{* *}$ \\
\hline HS27 & $0,31^{* *}$ & $3.870,68^{* *}$ & $2.604,65^{* *}$ & $16,07^{* *}$ & $-0,07^{\text {n.s }}$ & $0,40^{* *}$ & $2,39^{* *}$ \\
\hline HS28 & $0,31^{* *}$ & $1.815,54^{* *}$ & $292,15^{* *}$ & $0,25^{* *}$ & $1,18^{* *}$ & $0,01^{* *}$ & $1,44^{* *}$ \\
\hline L33 & $-0,37^{* *}$ & $-246,37^{\text {n.s }}$ & $-1.002,99^{*}$ & $-4,82^{\mathrm{n} . \mathrm{s}}$ & $-0,67^{* *}$ & $-0,33^{* *}$ & $0,44^{\mathrm{n} . \mathrm{s}}$ \\
\hline HS34 & $0,18^{* *}$ & $1.548,17^{*}$ & $544,13^{\text {n.s }}$ & $7,95^{* *}$ & $0,51^{\mathrm{n} . \mathrm{s}}$ & $0,12^{\mathrm{n} . \mathrm{s}}$ & $1,58^{* *}$ \\
\hline HS35 & $0,15^{*}$ & $-377,40^{\text {n.s }}$ & $-618,92^{\text {n.s }}$ & $-7,01^{*}$ & $0,46^{\mathrm{n} . \mathrm{s}}$ & $0,01^{\mathrm{n} . \mathrm{s}}$ & $-0,48^{\text {n.s }}$ \\
\hline HS36 & $0,23^{* *}$ & $476,64^{\mathrm{n} . \mathrm{s}}$ & $-45,04^{\mathrm{n} . \mathrm{s}}$ & $0,01^{\mathrm{n} . \mathrm{s}}$ & $0,41^{\mathrm{n} . \mathrm{s}}$ & $0,21^{\mathrm{n} . \mathrm{s}}$ & $0,27^{n . s}$ \\
\hline HS37 & $0,18^{*}$ & $911,85^{\text {n.s }}$ & $1.310,39^{* *}$ & $7,44^{*}$ & $1,03^{* *}$ & $0,16^{\mathrm{n} . \mathrm{s}}$ & $0,84^{\mathrm{n} . \mathrm{s}}$ \\
\hline HS38 & $-0,19^{n \cdot s}$ & $-4.680,81^{* *}$ & $-1.236,67^{* *}$ & $-4,15^{* *}$ & $-1,22^{* *}$ & $-0,45^{* *}$ & $-3,81^{* *}$ \\
\hline L44 & $-0,51^{* *}$ & $-7.335,34^{* *}$ & $-5.717,84^{* *}$ & $-24,34^{* *}$ & $0,18^{\mathrm{n} \cdot \mathrm{s}}$ & $-0,61^{* *}$ & $-5,55^{* *}$ \\
\hline HS45 & $0,12^{\mathrm{n} . \mathrm{s}}$ & $2.618,84^{* *}$ & $1.828,96^{* *}$ & $4,77^{\mathrm{n} . \mathrm{s}}$ & $0,13^{n . s}$ & $0,00^{\mathrm{n} . \mathrm{s}}$ & $2,05^{* *}$ \\
\hline HS46 & $0,1^{\mathrm{n} . \mathrm{s}}$ & $1.161,00^{\mathrm{n} . \mathrm{s}}$ & $1.639,92^{* *}$ & $18,09 * *$ & $0,08^{\mathrm{n} \cdot \mathrm{s}}$ & $0,34^{* *}$ & $1,36^{* *}$ \\
\hline HS47 & $0,1^{\mathrm{n} . \mathrm{s}}$ & $20,40^{\text {n.s }}$ & $786,59^{\text {n.s }}$ & $11,48^{* *}$ & $0,21^{\mathrm{n} . \mathrm{s}}$ & $0,10^{\mathrm{n} . \mathrm{s}}$ & $0,90^{\text {n.s }}$ \\
\hline HS48 & $0,3^{*}$ & $5.175,78^{* *}$ & $3.064,69^{* *}$ & $10,91^{* *}$ & $-0,04^{\mathrm{n} . \mathrm{s}}$ & $0,41^{\mathrm{n} . \mathrm{s}}$ & $1,92^{* *}$ \\
\hline L55 & $-0,31^{* *}$ & $-4.836,24^{* *}$ & $-3.951,53^{* *}$ & $-14,29^{* *}$ & $-0,67^{* *}$ & $-0,48^{* *}$ & $-2,32 * *$ \\
\hline HS56 & $0,05^{\mathrm{n} . \mathrm{s}}$ & $2.360,57^{* *}$ & $1.591,58^{* *}$ & $12,23^{* *}$ & $0,78^{* *}$ & $0,21^{\mathrm{n} . \mathrm{s}}$ & $0,82^{\text {n.s }}$ \\
\hline HS57 & $0,09^{\mathrm{n} . \mathrm{s}}$ & $980,54^{\mathrm{n} . \mathrm{s}}$ & $893,24^{\text {n.s }}$ & $1,90^{\mathrm{n} . \mathrm{s}}$ & $-0,09^{\mathrm{n} . \mathrm{s}}$ & $0,03^{n . s}$ & $0,24^{\mathrm{n} . \mathrm{s}}$ \\
\hline HS58 & $0,18^{\mathrm{n} . \mathrm{s}}$ & $921,82^{* *}$ & $651,99 * *$ & $4,36^{* *}$ & $0,91^{\mathrm{n} . \mathrm{s}}$ & $0,05^{* *}$ & $0,18^{* *}$ \\
\hline L66 & $-0,49^{* *}$ & $-5.346,72^{* *}$ & $-3.576,72^{* *}$ & $-19,99^{* *}$ & $-0,52^{*}$ & $-0,06^{* *}$ & $-3,45^{* *}$ \\
\hline HS67 & $0,019^{n . s}$ & $1.452,53^{*}$ & $330,82^{n . s}$ & $-7,10^{*}$ & $-0,39^{\mathrm{n} . \mathrm{s}}$ & $0,13^{\mathrm{n} . \mathrm{s}}$ & $0,91^{\mathrm{n} . \mathrm{s}}$ \\
\hline HS68 & $0,158^{* *}$ & $2.274,04^{* *}$ & $1.300,35^{* *}$ & $6,10^{* *}$ & $0,11^{\mathrm{n} . \mathrm{s}}$ & $0,24^{\text {n.s }}$ & $1,13^{* *}$ \\
\hline L77 & $-0,42^{* *}$ & $-4.548,89^{* *}$ & $-4.216,54^{* *}$ & $-17,20^{* *}$ & $-0,27^{n . s}$ & $-0,43^{* *}$ & $-3,41^{* *}$ \\
\hline HS78 & $0,14^{* *}$ & $924,34^{* *}$ & $1.147,17^{* *}$ & $0,77^{* *}$ & $-0,52^{*}$ & $0,07^{\mathrm{n} . \mathrm{s}}$ & $1,19^{* *}$ \\
\hline L88 & $-0,42^{* *}$ & $-4.221,98^{* *}$ & $-2.652,31^{* *}$ & $-10,58^{* *}$ & $-0,77^{\mathrm{n} \cdot \mathrm{s}}$ & $-0,34^{* *}$ & $-0,86^{* *}$ \\
\hline
\end{tabular}

${ }^{*} \mathrm{e} * *$ - significativo a $5 \%$ e a $1 \%$ de probabilidade respectivamente pelo teste t. ${ }^{\text {n.s. }}$ : não significativo. 


\section{CONCLUSÕES}

1. Os efeitos não aditivos foram mais importantes que os efeitos aditivos para a variação dos genótipos para as características PEE, PEC, ALT, MASSA, COR, DIAM e COMP, o que possibilita inferir que a heterose é importante na seleção de híbridos para a produção de milho verde.

2. A linhagem L55 com maior concentração de alelos favoráveis para o incremento das características PEC, PEE, MASSA, COR e COMP, é a mais indicada para a formação de novos híbridos de milho verde.

3. As combinações L22xL77 (HS27), L44xL88 (HS48) e L22xL44 (HS24) permitiram maior efeito heterótico e média alta para as características PEE, PEC e COMP, simultaneamente, sendo estes híbridos mais indicados ao mercado de milho verde in natura.

\section{AGRADECIMENTOS}

Os autores agradecem à FAPEMIG (Fundação de Amparo à Pesquisa do Estado de Minas Gerais www.fapemig.br) pelo auxílio financeiro no desenvolvimento do trabalho.

\section{REFERÊNCIAS}

ALBUQUERQUE, C. J. B. Desempenho de híbridos de milho verde na região Sul de Minas Gerais. 2005. 56 p. (Mestrado em Fitotecnia) - Universidade Federal de Lavras, Lavras, MG.

BORDALLO, P. N.; PEREIRA, M. G.; AMARAL JÚNIOR, A. T.; GABRIEL, A. P. C. Análise dialélica de genótipos de milho doce e comum para caracteres agronômicos e proteína total. Horticultura Brasileira, Brasília, v. 23, n. 1, p. 123-127, jan./ mar. 2005.

BOTTINI, P. R.; TSUNECHIRO, A.; COSTA, F. A. G. da. Viabilidade da produção de milho verde na "safrinha". Informações Econômicas, São Paulo, v. 25, n. 3, p. 49-53, mar. 1995.

CARDOSO, M. J.; CARVALHO, H. W. L.; RIVEIRO, V. Q. Avaliação preliminar de variedades de milho para produção de espiga verde em sistema agrícola familiar. In: REUNIÃO ANUAL DA SOCIEDADE INTERAMERICANO DE HORTICULTURA TROPICAL, 49., 2003, Fortaleza. Resumos... Fortaleza: Embrapa Agroindústria Tropical, 2003. p. 72. (Documentos, 67)

CRUZ, C. D. Programa Genes. Aplicativo computacional em genética e estatística. Viçosa. MG: UFV, 1997. 442 p.

CRUZ, J. C.; PEREIRA FILHO, I. A. Cultivares de Milho disponíveis no mercado de sementes do Brasil para a safra 2006/07. 2007. Disponível em < http:// www.cnpms.embrapa.br/milho/cultivares/index.php>. Acesso em: 21 de jan. 2007.
GRIFFING, B. A. Concept of general and specific combining ability in relation to diallel crossing systems. Australian Journal Biology Science, East Melbourn, v. 9, p. 463-493, 1956.

LEMOS, M. A.; GAMA, E. E. G.; MENEZES, D.; SANTOS, V. F.; TABOSA, J. N. Avaliação de dez linhagens e seus híbridos de milho superdoce em um dialelo completo. Horticultura Brasileira, Brasília, v. 20, n. 2, p. 167-170, jun. 2002.

MATOS, M. J. L. F.; TAVARES, S. A.;SANTOS, F. F. DOS; MELO, M. F. DE; LANA, M. M. Milho verde. 2006. Disponível em: < h t t p : / / w w w.c n ph.embrapa.br/paginas / dicas_ao_consumidor/milho_verde.htm>. Acesso em: 16 de jun. 2006.

MONTEIRO, M. A. R.; PEREIRA FILHO, I. A.; GAMA, E. E. G.; KARAM, D.; CRUZ, J. C. Avaliação Preliminar de Híbridos Triplos de Milho Visando Consumo Verde. In: CONGRESSO NACIONAL DE MILHO E SORGO, 24., 2002, Florianópolis. Anais... Florianópolis, 2002. 1CD-ROM

OLIVEIRA, L, A. A. de; GROSZMAN, A.; COSTA, R. A. da. Caracteres da espiga de cultivares de milho no estádio verde. Pesquisa Agropecuária Brasileira, Brasília, v. 22, n. 6, p. 587592, jun. 1987.

PEREIRA FILHO, I. A.; CRUZ, J. C. GAMA, E. E. G. Cultivares para o consumo verde. In: PEREIRA FILHO, I. A. (Ed.). O cultivo do milho verde. Brasília. DF: EMBRAPA informação tecnológica, 2003. 204 p.

SAWAZAKI, E.; POMMER,. C. V.; ISHIMURA, I. Avaliação de cultivares de milho para utilização no estádio de verde. Ciência e Cultura, Campinas, v. 31, n. 11, p. 1297-1302, nov. 1979.

SCAPIM, C. A.; CRUZ, C. D.; ARAÚJO, J. M. Cruzamentos dialélicos entre sete cultivares de milho doce. Horticultura Brasileira, Brasília, v. 13, n. 1, p. 19-21, maio 1995.

SCOTT, A. J.; KNOTT, M. A cluster analysis method for grouping means in the analysis of variance. Biometrics, Washington, v. 30, n.3, p. 507-512, Sept. 1974.

TABOSA,J.N.;OLIVEIRA, J.P.;REIS. A. R. M. B.;AZEVEDONETO, A. D.; MONTEIRO, M. C. D.; FERREIRA, P.F. Avaliação preliminar de cultivares para produção de milho verde na Zona da Mata Norte de Pernambuco. In: CONGRESSO NACIONAL DE MILHO E SORGO, 23., 2000. Uberlândia. MG. A inovação tecnológica e a competitividade no contexto dos mercados globalizados. Resumos Expandidos... Sete Lagos: ABMS/Embrapa Milho e Sorgo/ Universidade Federal de Uberlândia, 2000. 1CD-ROM.

TEIXEIRA, F. F.; SOUZA, R. P. de; GAMA, E. E. G.; PACHECO, C. A. P.; PARENTONI, S. N.; SANTOS, MX. dos; MEIRELLES, W. F. Ciência e Agrotecnologia, Lavras, v. 25, n. 3, p. 483-488, maio/jun. 2001.

VALENTINI, L.; SHIMOYA, A. Comportamento de cultivares de milho verde em Campos dos Goytacazes- Região Norte Fluminense. In: CONGRESSO NACIONAL DE MILHO E SORGO, 22., 1998, Recife, PE. Globalização e segurança alimentar. Resumos expandidos... Recife: ABMS, 1998. (1CD-ROM) 